\title{
Correction to: Radiation Safety in Videofluoroscopic Swallowing Study: Systematic Review
}

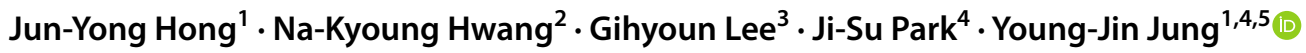

Published online: 5 November 2020

๑) Springer Science+Business Media, LLC, part of Springer Nature 2020

\section{Correction to: Dysphagia \\ https://doi.org/10.1007/s00455-020-10112-3}

The original version of this article unfortunately contained a mistake. The presentation of Fig. 1 was incorrect. The correct version of Fig. 1 is given below.

The original article can be found online at https://doi.org/10.1007/ s00455-020-10112-3.

Ji-Su Park

jisu627@hanmail.net

$\triangle$ Young-Jin Jung

microbme@dongseo.ac.kr

1 Department of Multidisplinary Radiological Science, Graduate School, DongSeo University, 47 Jurye-ro, Sasang-gu, Busan 47011, Republic of Korea

2 Department of Occupational Therapy, Seoul North Municipal Hospital, Seoul, Republic of Korea

3 Department of Physical and Rehabilitation Medicine, Center for Prevention and Rehabilitation, Heart Vascular Stroke Institute, Samsung Medical Center, Sungkyunkwan University School of Medicine, 81 Irwon-ro, Gangnam-gu, Seoul 06351, Republic of Korea

4 Advanced Human Resource Development Project Group for Health Care in Aging Friendly Industry, Dongseo University, 47 Jurye-ro, Sasang-gu, Busan 47011, Republic of Korea

5 Department of Radiological Science at Health Sciences Division in DongSeo University, 47 Jurye-ro, Sasang-gu, Busan 47011, Republic of Korea 
Fig. 1 A PRISMA flow diagram

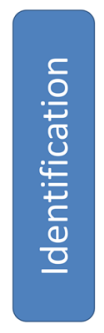

Materials identified through
database searching
$(n=2,805)$
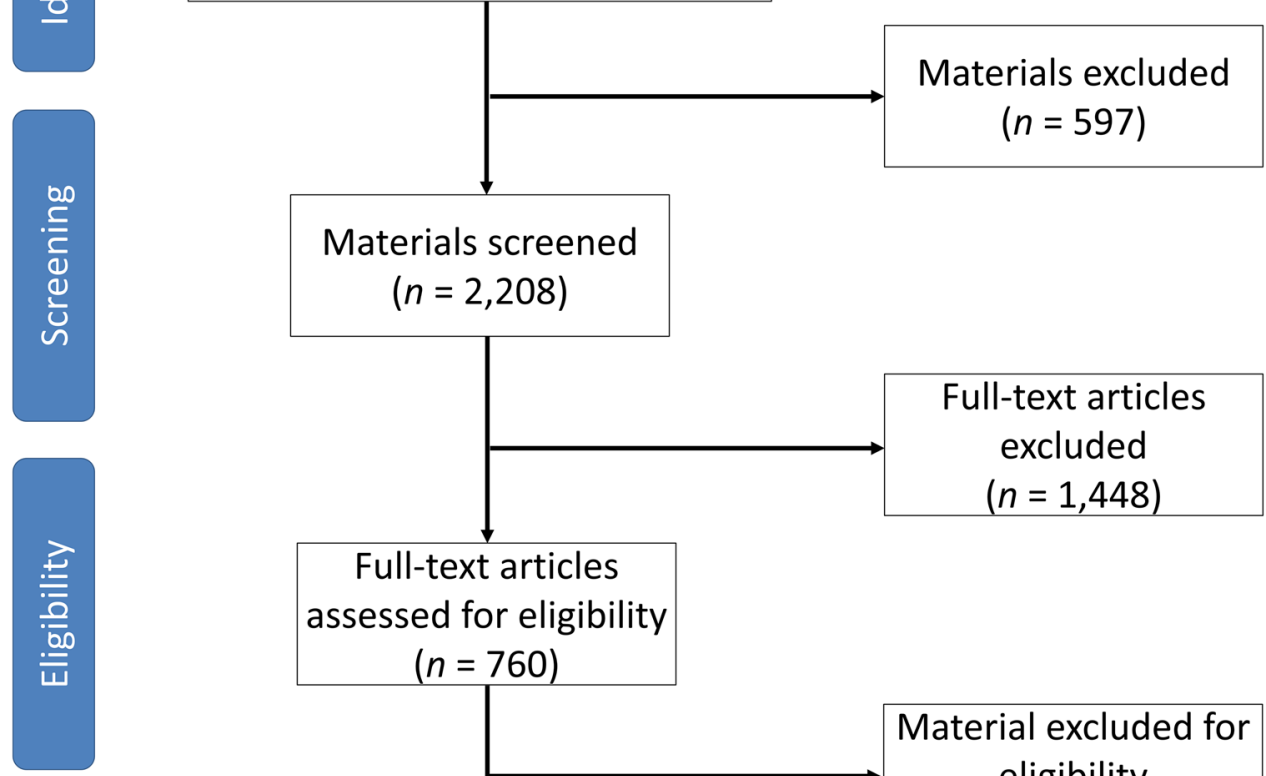

Full-text articles assessed for eligibility $(n=760)$

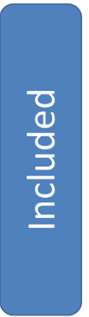

Material excluded for eligibility $(n=747)$

Materials selected for review

$(n=13)$

Publisher's Note Springer Nature remains neutral with regard to jurisdictional claims in published maps and institutional affiliations. 